\title{
A Low-Cost Noise Wave Correlator for Noise Parameters Extraction by Cold Noise Power Measurement
}

\author{
Abdul-Rahman Ahmed ${ }^{\mathrm{a}}$, Kyung-Whan Yeom $^{\mathrm{b}}$ \\ ${ }^{a}$ Department of Electrical/Electronic Eng. KNUST, Kumasi, Ghana \\ ${ }^{b}$ Deparment of Radio Science an Engineering d, Chungnam Nat. Univ., Daejeon, South Korea
}

\begin{abstract}
Conventionally, the noise parameters of a Device under Test (DUT) which generally characterize the noise performance of the DUT, are obtained via the impedance tuner technique. The authors have previously presented a technique which eliminates the need for impedance tuner, and rather employs an 8-port network that enables the extraction of the noise correlation matrix of a given DUT and thus its noise parameters. In this paper, we present a further simplification of the 8-port network technique, which also eliminates the need for a conventional external noise source. Cold noise powers emanating from a DUT are measured via a 6-port network with the aid of matched termination. The measured noise powers provide sufficient information for determining the noise wave correlation matrix of a DUT, which are then converted into the conventional 2-port noise parameters. The proposed technique is simple, fast and is verified to give a good estimation of the noise parameters of selected DUTs.
\end{abstract}

Index Terms: 6-port network, noise parameters, noise waves, noise correlation matrix, calibration, noise figure analyzer.

(C) 2016 Published by MECS Publisher. Selection and/or peer review under responsibility of the Research Association of Modern Education and Computer Science

\section{Introduction}

The noise figure represents the noise contribution of a 2-port device to an output load compared with an external noise source having a fixed source impedance and at temperature $T_{o}$ [1]. The noise figure varies with varying source impedance [2] due to the dependence of an equivalent Norton current source (representing the noise of a DUT) on the source impedance. The noise parameters, which characterize the noise performance of a Device under Test (DUT) and which are crucial in the design of low noise amplifiers (LNAs), describe the dependence of the noise figure on the source impedance of the DUT; a fact that is exploited in the measurement

* Corresponding author. Tel.: 09988292611;

E-mail address: 
of the noise parameters in the Multi-Impedance Technique [3].

In the Multi-Impedance Technique, a number of noise figure measurements corresponding to different source impedance of the DUT, are made, following which the minimum noise figure and the optimum reflection coefficients, at which the former occurs, are obtained. Since there are four noise parameters (four unknowns), theoretically, only four measurements are required to determine the noise parameters. It has

however been observed that [3] this technique is not quite satisfactory; the main reason being that, the partial derivative of the noise factor, with respect to source admittance is zero at the minimum noise factor. It has also been observed [4] that, this technique is highly sensitive to measurement error especially for devices having large values of $R_{n}(>150 \Omega$ ). It was further observed that, [5] the least-square fit approach as proposed in [3] sometimes leads to meaningless results. Other variations of the Multi-Impedance technique that improves the accuracy of the extracted noise parameters have been reported in [6]-[11], [12].

The use of the bulky impedance tuner in the multi-impedance technique has some drawbacks including making the measurement less convenient. Long measurement time and repeatability errors are some of the other well-known drawbacks of the impedance tuner technique [13]. The bulky nature of the impedance tuner also complicates on-wafer measurement. A number of alternative techniques based on the wave representation of the noisy 2-port and which avoids the use of microwave impedance tuners have therefore emerged.

These techniques have proven effective due to the convenience of expressing the relationship between the waves in terms of scattering parameters. Once the noise wave parameters and corresponding correlation matrix for a given DUT are extracted, transformations already exist for converting the noise wave correlation matrix to noise parameters.

The analysis of linear noisy networks based on the noise wave formalism abound in the literature [14], [15], [16], [17] - [20] and form the basis for some of the noise parameters extraction techniques discussed below.

Although not many, the techniques appearing in [14], [16]-[22], [23]-[26] are significant contributions as far as the noise wave technique for the extraction of noise parameters is concerned. In one of the early works in this regard [14], an almost lossless source of varying phases and a matched load enabled the determination of the noise temperatures of three sets of wave parameters in addition to the phase of the cross-correlated noise power spectrum, that completely characterize the noise properties of a linear two-port.

In yet another significant contribution [15], [20]-[21], the self-correlated noise power densities of the DUT were measured via a thru and the cross-correlated noise power densities via a $3 \mathrm{~dB}$ hybrid coupler, alternately connected to the noise source and DUT via two sets of circulators, which resulted in the noise wave parameters of the DUT. It must also be mentioned that, the use of a 6-port network [27], was seen as an alternative [20] to the use of couplers/Thru combination, although the implementation of same as a practical noise wave correlator was deferred to future work.

The authors presented a technique [28] for noise parameters extraction that makes use of an 8-port extension of the 6-port network. The necessity for the 8-port extension arose from the need to allow for simultaneous connection of an external noise source and the DUT during measurement.

The present method recognizes that, by directly measuring the noise powers at the output of the 6-port network, as opposed to the case in the previous method, where noise figure and gain measurement were used to determine the noise powers emanating from the 8-port network, the external noise source may not be necessary. Thus, the extension to an 8-port network to cater for the external noise source, resulting in complex calibration process, may not be necessary after all. Elimination of the external noise source and using a standard $50 \Omega$ termination instead for cold noise power measurement means a 6-port network works just fine as a noise wave correlator. A new formulation for the estimation of the noise powers exiting the measurement ports of the 6port network is developed, leading to new simplified calibration equations and procedures and thus simplified technique for the determination of the noise wave parameters of the DUT. The proposed technique is experimentally verified to give a good estimation of the noise parameters of a $10 \mathrm{~dB}$ attenuator, as sample DUT, and compares very favorably with the simulated noise parameters of the same DUT. 


\section{Formulation}

Figure 1 shows the 6-port network, consisting of five (5) Wilkinson Power Dividers and one (1) $90^{\circ}$ Hybrid. The noise waves emanating from the DUT, $c_{d l}$ and $c_{d 2}$, denoted as vector $\mathbf{c}_{D}$, are correlated via the 6-port network through the input ports (ports 5 and 6) and exit the output ports (ports 1-4) through the SP4T switch, together with the noise waves emanating from the 6-port network and associated terminations. It is obvious from the structure of the 6-port network that, the noise wave $c_{d 1}$ and $c_{d 2}$ can be determined from noise powers measured at ports 1 and 2 respectively. Information on the correlations between $c_{d 1}$ and $c_{d 2}$ on the other hand can be determined from the noise powers emerging from ports 3 and 4, which give the quadrature and in-phase sum of the input noise waves $c_{d 1}$ and $c_{d 2}$ respectively.

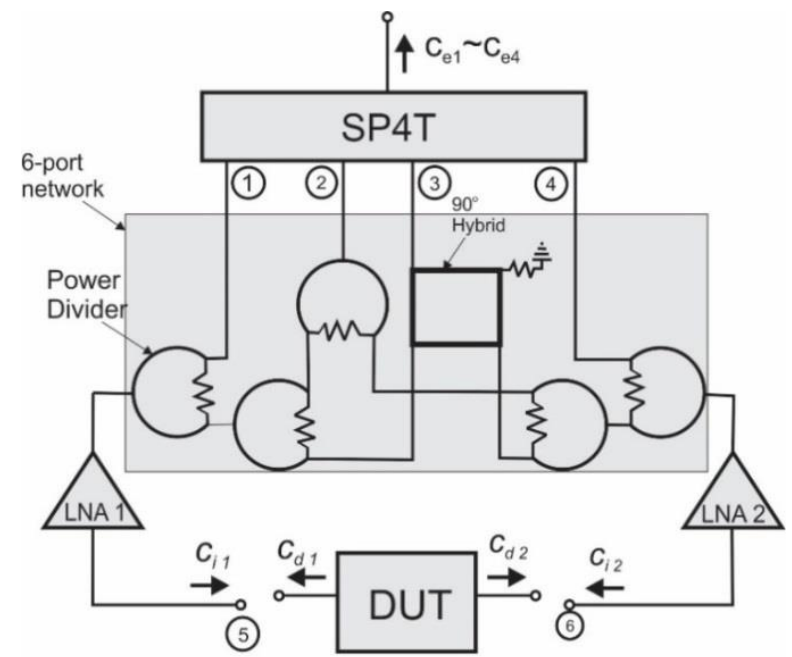

Fig.1. The 6-port network and associated noise waves

The S-parameters of the 6-port network can be partitioned into four (4) sub matrices from which the relationship between input and output parameters can be expressed as in (1). $\mathbf{S}_{i j}$ in (1) represents the partitioned S-parameters of the 6-port network. $\mathbf{b}$ is noise waves out of the 6-port, $\mathbf{a}$ is noise wave input from port and switch terminations and $\mathbf{c}$ is the internal noise of the 6-port network. The subscript $e$, represents the output ports (ports 1-4) and $i$ represent the DUT noise input ports (ports 5 and 6) respectively.

$$
\mathbf{b}=\left(\begin{array}{l}
\mathbf{b}_{e} \\
\mathbf{b}_{i}
\end{array}\right)=\left(\begin{array}{ll}
\mathbf{S}_{e e} & \mathbf{S}_{e i} \\
\mathbf{S}_{i e} & \mathbf{S}_{i i}
\end{array}\right)\left(\begin{array}{l}
\mathbf{a}_{e} \\
\mathbf{a}_{i}
\end{array}\right)+\left(\begin{array}{c}
\mathbf{c}_{e} \\
\mathbf{c}_{i}
\end{array}\right)
$$

Expanding (1),

$$
\begin{aligned}
& \mathbf{b}_{e}=\mathbf{S}_{e e} \mathbf{a}_{e}+\mathbf{S}_{e i} \mathbf{a}_{i}+\mathbf{c}_{e} \\
& \mathbf{b}_{i}=\mathbf{S}_{i e} \mathbf{a}_{e}+\mathbf{S}_{i i} \mathbf{a}_{i}+\mathbf{c}_{i}
\end{aligned}
$$

Denoting the DUT S-parameters as $\mathbf{S}_{D}$, the noise waves delivered to the input of the 6-port network (ports 5 and 6) from the DUT is given by 


$$
\mathbf{b}_{D}=\mathbf{S}_{D} \mathbf{a}_{D}+\mathbf{c}_{D}
$$

The noise emanating from the 2-port DUT is incident on the input ports of the 6-port network and thus $\mathbf{b}_{D}=\mathbf{a}_{i}$ and the noise emanating from the 6-port network and appearing at its input port is also incident on the 2-port DUT. Thus $\mathbf{a}_{D}=\mathbf{b}_{i}$. Therefore,

$$
\mathbf{a}_{i}=\mathbf{S}_{D} \mathbf{b}_{i}+\mathbf{c}_{D}
$$

Expressing this in terms of $\mathbf{b}_{i}$

$$
\mathbf{b}_{i}=\mathbf{S}_{D}^{-1} \mathbf{a}_{i}-\mathbf{S}_{D}^{-1} \mathbf{c}_{D}
$$

and substituting (6) into (3) results in

$$
\mathbf{S}_{D}^{-1} \mathbf{a}_{i}-\mathbf{S}_{D}^{-1} \mathbf{c}_{D}=\mathbf{S}_{i e} \mathbf{a}_{e}+\mathbf{S}_{i i} \mathbf{a}_{i}+\mathbf{c}_{i}
$$

Hence

$$
\left(\mathbf{I}-\mathbf{S}_{i i} \mathbf{S}_{D}\right) \mathbf{a}_{i}=\mathbf{S}_{D} \mathbf{S}_{i e} \mathbf{a}_{e}+\mathbf{S}_{D} \mathbf{c}_{i}+\mathbf{c}_{D}
$$

Therefore,

$$
\mathbf{a}_{i}=\left(\mathbf{I}-\mathbf{S}_{i i} \mathbf{S}_{D}\right)^{-1} \mathbf{S}_{D} \mathbf{S}_{i e} \mathbf{a}_{e}+\left(\mathbf{I}-\mathbf{S}_{i i} \mathbf{S}_{D}\right)^{-1} \mathbf{S}_{D} \mathbf{c}_{i}+\left(\mathbf{I}-\mathbf{S}_{i i} \mathbf{S}_{D}\right)^{-1} \mathbf{c}_{D}
$$

Finally, substituting (9) into (2)

$$
\mathbf{b}_{e}=\mathbf{S}_{e e} \mathbf{a}_{e}+\mathbf{S}_{e i}\left\{\left(\mathbf{I}-\mathbf{S}_{i i} \mathbf{S}_{D}\right)^{-1} \mathbf{S}_{D} \mathbf{S}_{i e} \mathbf{a}_{e}+\left(\mathbf{I}-\mathbf{S}_{i i} \mathbf{S}_{D}\right)^{-1} \mathbf{S}_{D} \mathbf{c}_{i}+\left(\mathbf{I}-\mathbf{S}_{i i} \mathbf{S}_{D}\right)^{-1} \mathbf{c}_{D}\right\}+\mathbf{c}_{e}
$$

Thus,

$$
\mathbf{b}_{e}=\mathbf{S}_{e e} \mathbf{a}_{e}+\Lambda \mathbf{S}_{D} \mathbf{S}_{i e} \mathbf{a}_{e}+\Lambda \mathbf{S}_{D} \mathbf{c}_{i}+\Lambda \mathbf{c}_{D}+\mathbf{c}_{e}
$$

Defining new embedded matrices, equation (11) can be expressed as

$$
\mathbf{b}_{e}=\mathbf{M a} \mathbf{a}_{e}+\mathbf{Q} \mathbf{c}_{i}+\Lambda \mathbf{c}_{D}+\mathbf{c}_{e}
$$

Each matrix is then defined as

$$
\mathbf{\Lambda}=\mathbf{S}_{e i}\left(\mathbf{I}-\mathbf{S}_{i i} \mathbf{S}_{D}\right)^{-1}
$$




$$
\begin{aligned}
& \mathbf{M}=\mathbf{S}_{e e}+\boldsymbol{\Lambda} \mathbf{S}_{D} \mathbf{S}_{i e} \\
& \mathbf{Q}=\boldsymbol{\Lambda} \mathbf{S}_{D}
\end{aligned}
$$

Equation (12) represents the relationship between possible noise sources and the noise waves emanating at the output ports of the 6-port network. Suppose the internal noise sources of the 6-port network, and any external noise source resulting from terminations are accounted for through calibration, then, $\mathbf{c}_{e}=\mathbf{c}_{i}=0$ and $\mathbf{a}_{e}=0$. From (10), the output noise waves emanating from the four (4) output ports of the 6-port network which is due solely to the DUT, $\boldsymbol{\beta}_{e}$ can then be related to the actual noise waves of the DUT $\mathbf{c}_{D}$ as,

$$
\boldsymbol{\beta}_{e}=\Lambda \mathbf{c}_{D}
$$

Thus, the noise waves parameters of the DUT $\left(\left|c_{d 1}\right|^{2}, c_{d 2} c_{d 1}^{*}, c_{d 1} c_{d 2}^{*},\left|c_{d 2}\right|^{2}\right)$ can be determined and hence its $2 \times 2$ chain matrix representation of the noise correlation matrix; from which the noise parameters can be computed as [29]

$$
\begin{aligned}
& R_{n}=\frac{c_{11}}{4 k T} \\
& Y_{o p t}=\sqrt{\frac{c_{22}}{c_{11}}-\left\{\operatorname{Im}\left(\frac{c_{12}}{c_{11}}\right)\right\}^{2}}+j \operatorname{Im}\left(\frac{c_{12}}{c_{11}}\right) \\
& N F_{\text {min }}=1+\frac{c_{12}+c_{11} Y_{o p t}^{*}}{2 k T}
\end{aligned}
$$

where $c_{i j}$ are the elements of the ABCD noise correlation matrix obtained from the transformation of the noise wave parameters, $k$ is Boltzmann's constant and $T$ is temperature.

\section{Calibration}

Since the noise wave spectral densities emerging at the output of the 6-port network include the noise waves from terminations in the SP4T switch as well as terminations at ports 5 and 6 that are employed for calibration and the noise waves emanating from within the 6-port itself, it is necessary to determine these noise waves and their respective contributions to the output noise power. This process, termed calibration, enables us to determine the noise waves exclusively due to the DUT. Referring to Fig. 1, the noise waves of the DUT $c_{d l}$ and $c_{d 2}$ combine with that from the 6-port network, switch terminations (ports 1-4) as well as standard terminations at ports 5 and 6, and appear at the output ports of the 6-port network. Calibration of the 6-port network is thus necessary in determining the noise waves exclusively due to the DUT.

The calibration follows from equation (12). In the absence of DUT, the input ports of the 6-port network (ports 5 and 6) are terminated in standard $50 \Omega$ terminations. The output noise wave vector $\mathbf{b}_{e}$ for this situation will be due to the thermal noise from the $50 \Omega$ terminations, switch terminations as well as the noise internal to the 6-port network which appear at the output ports. The thermal noise from switch termination, $\mathbf{a}_{e}$ in (2) can be determined from their available noise power density, $k T_{o}$ and thus the internal noise emanating from the 6-port 
network, $\mathbf{c}_{e}$ can thus be determined. Note that, the noise wave vector $\mathbf{a}_{i}$ is terminated in the matched $50 \Omega$ termination and since the DUT in this case is a pair of $50 \Omega$ terminations connected to the input ports, $\mathbf{S}_{D}$ is zero and (2) reduces to

$$
\mathbf{b}_{e T}=\mathbf{S}_{e e} \mathbf{a}_{e}+\mathbf{c}_{e}
$$

where $\mathbf{b}_{e T}$ is the noise waves available at the output ports when the input ports are terminated in matched $50 \Omega$ terminations. The noise powers at the output ports in this case can be expressed as

$$
d\left(\mathbf{b}_{e T} \mathbf{b}_{e T}^{\dagger}\right)=d\left\{\left(\mathbf{S}_{e e} \mathbf{a}_{e}+\mathbf{c}_{e}\right) \times\left(\mathbf{S}_{e e} \mathbf{a}_{e}+\mathbf{c}_{e}\right)^{\dagger}\right\}=d\left\{\left(\mathbf{S}_{e e} \mathbf{a}_{e} \mathbf{a}_{e}^{\dagger} \mathbf{S}_{e e}^{\dagger}\right)+\mathbf{c}_{e} \mathbf{c}_{e}^{\dagger}\right\}=d\left\{\left(\mathbf{S}_{e e} \mathbf{S}_{e e}^{\dagger}\right) k T_{o}+\mathbf{C}_{e e} k T_{o}\right\}
$$

The operation $d\{\bullet\}$ denotes the diagonal of a matrix and $\dagger$ represents conjugate transpose of a matrix. $\mathbf{C}_{e e}$ in (16) is the correlation matrix of the noise waves emanating from the 6-port network, normalized to thermal noise power, and appearing at the output ports. Thus, $\mathbf{C}_{e e}$ can be determined from

$$
d\left(\mathbf{C}_{e e}\right)=d\left(\mathbf{b}_{e T} \mathbf{b}_{e T}^{\dagger}\right)-d\left(\mathbf{S}_{e e} \mathbf{S}_{e e}^{\dagger}\right)
$$

Referring to Fig. $1, c_{i 1}, c_{i 2}$, and their correlation with $\mathbf{c}_{e}$ can be determined by connecting highly reflective loads at ports 5 and 6 and observing the changes in $\mathbf{c}_{e}$ at ports 1-4. Three terminations such as open, short and offset open are connected to port 5 to determine $c_{i 1}$ and its correlation with $\mathbf{c}_{e}$ while port 6 is terminated in 50 ohm load. Alternately, $c_{i 2}$ and its correlation with $\mathbf{c}_{e}$ can be obtained by changing the roles of ports 5 and 6 . Denoting the measured noise wave for this case, normalized by the thermal noise, $k T_{o}$, as $\mathbf{n}_{C}$, the following relation can be obtained using (12).

$$
d\left\{\mathbf{b}_{e} \mathbf{b}_{e}^{\dagger}\right\}=k T_{o} \mathbf{n}_{C}=d\left\{\left(\mathbf{M} \mathbf{a}_{e}+\mathbf{Q} \mathbf{c}_{i}+\mathbf{c}_{e}\right)\left(\mathbf{M} \mathbf{a}_{e}+\mathbf{Q} \mathbf{c}_{i}+\mathbf{c}_{e}\right)^{\dagger}\right\}+d\left(\mathbf{\Lambda} \mathbf{C}_{T} \mathbf{\Lambda}^{\dagger}\right)
$$

In (18), $\mathbf{C}_{T}$ represents the noise wave contribution to $\mathbf{n}_{c}$ by the $50 \Omega$ termination at port 5 or 6 , depending on which port the reflective load is connected to. $\mathbf{C}_{T}$ for the matched $50 \mathrm{ohm}$ termination is given by

$$
\mathbf{C}_{T}=\left(\begin{array}{ll}
0 & 0 \\
0 & 1
\end{array}\right)
$$

Further simplification of (18) results in

$$
\mathbf{n}_{C}=d\left\{\mathbf{C}_{e e}+\mathbf{M} \mathbf{M}^{\dagger}\right\}+d\left(\mathbf{\Lambda} \mathbf{C}_{T} \mathbf{\Lambda}^{\dagger}\right)+d\left(\mathbf{Q} \mathbf{C}_{i e}+\mathbf{C}_{e i} \mathbf{Q}^{\dagger}+\mathbf{Q} \mathbf{C}_{i i} \mathbf{Q}^{\dagger}\right)
$$

Note that the first 2 terms on the right hand side of (19) are known when the DUT and 6-port S-parameter are known. Defining excess noise due to correlation, normalized by $k T_{o}$, as $\boldsymbol{\beta}_{C}$, and arranging the known terms on the left hand side and the unknown terms on the right hand side results in 


$$
\boldsymbol{\beta}_{C}=\mathbf{n}_{C}-d\left\{\mathbf{C}_{e e}+\mathbf{M} \mathbf{M}^{\dagger}\right\}-d\left(\mathbf{\Lambda} \mathbf{C}_{T} \mathbf{\Lambda}^{\dagger}\right)=d\left(\mathbf{Q} \mathbf{C}_{i e}+\mathbf{C}_{e i} \mathbf{Q}^{\dagger}+\mathbf{Q} \mathbf{C}_{i i} \mathbf{Q}^{\dagger}\right)
$$

$\mathbf{C}_{e e}$ has been determined in a previous calibration step, $\mathbf{M}, \boldsymbol{\Lambda}$ and $\mathbf{Q}$ are known from the S-parameters of the 6-port network and DUT, which in this case are the terminations at ports 5 and 6 . The unknowns are $\mathbf{C}_{i i}$, which are the normalized noise power spectrum from the 6-port network appearing at ports 5 and $6 ; \mathbf{C}_{i e}$ and $\mathbf{C}_{e i}$ are the normalized correlations between $\mathbf{c}_{i}$ and $\mathbf{c}_{e}$. These three quantities can be determined from three simultaneous equations generated using three different terminations such as open, short and offset open. Thus all the noise waves associated with the 6-port network and terminations would have been determined from these calibrations.

To measure the noise wave parameters of a DUT, the DUT is connected to the input ports, (ports 5 and 6) and because all the noise waves emanating from the 6-port network were determined a priori in the calibration process, the noise powers emanating from the DUT can easily be determined from

$$
\boldsymbol{\beta}_{D}=d\left(\boldsymbol{\Lambda} \mathbf{C}_{D D} \boldsymbol{\Lambda}^{\dagger}\right)=\mathbf{n}_{D}-d\left\{\mathbf{M} \mathbf{M}^{\dagger}+\left(\mathbf{C}_{e e}+\mathbf{Q} \mathbf{C}_{i e}+\mathbf{C}_{e i} \mathbf{Q}^{\dagger}+\mathbf{Q} \mathbf{C}_{i i} \mathbf{Q}^{\dagger}\right)\right\}
$$

where $\mathbf{n}_{D}$ is the measured noise power spectrum (normalized to thermal noise power) at the output of the 6-port network while the DUT is connected. Whereas $\mathbf{n}_{D}$ includes all the noise contributions from the 6-port network as well as terminations, $\boldsymbol{\beta}_{D}$ is the normalized excessive noise power due exclusively to the DUT. Since $\boldsymbol{\Lambda}$ is known from the S-parameters of the 6-port network, $\mathbf{C}_{D D}$, the normalized noise wave correlation matrix of the DUT can then be determined. As discussed in Section II, the noise parameters can then be determined from the noise correlation matrix.

\section{Measurement}

The measurement set-up is shown in Fig. 2. It consists of the 6-port network, two (2) sets of low noise amplifiers (LNAs), a single-pole four-throw (SP4T) switch, noise figure analyzer (NFA), D.C. Supply and computer connected via GPIB cable. The two (2) LNAs in Fig. 2 are for gain boosting and their DC voltages are supplied using a 6626A D.C supply from Agilent.

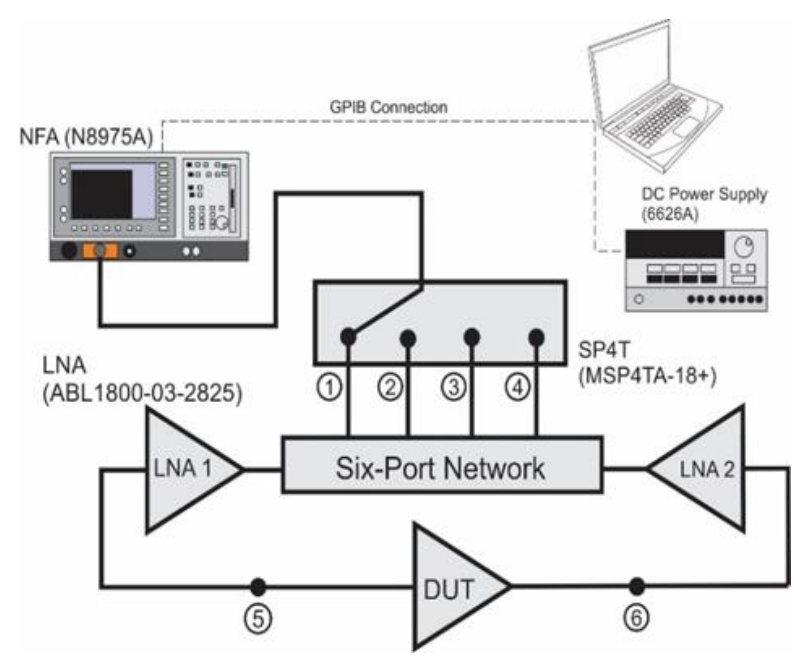

Fig.2. The 6-port network noise wave correlator measurement set up 
As noted previously, the essence of the calibration is to determine the noise contribution of the 6-port network to the measurement port as well as those of switch and standard $50 \Omega$ terminations. The following are the steps involved in the calibration. The reference to port numbers is as designated in Fig. 1.

Step1: Calibrate NFA with a standard $50 \Omega$ termination. The aim is to record as close to $0 \mathrm{~dB}$ noise powers as possible on the NFA.

Step2: The calibration set up is wired as shown in Fig. 2, but with different sets of terminations instead of the DUT.

This set of steps is for determining $\mathbf{c}_{e}$, the noise wave emitted by the 6-port network, directly into the measurement ports.

Step3: Two $50 \mathrm{ohm}$ terminations, are used to terminate ports 5 and 6.

Step4: Switching the output from port 1 to port 4, the noise waves emanating from the output of the 6-port network for this termination state is measured for each of the four measuring ports. These noise powers are then imported into the calibration equations to determine $\mathbf{c}_{e}$.

This set of steps is for determining $\mathbf{c}_{i}$, the noise wave emitted by the 6-port network into ports 5 and 6 , which are reflected and correlated with $\mathbf{c}_{e}$, and appear directly out of the measurement ports.

Step5: One of the $50 \mathrm{ohm}$ terminations is replaced with an open termination.

Step6: Steps 4 is repeated for this state of termination.

Step7: The open and $50 \mathrm{ohm}$ terminations at ports 5 and 6 are switched and step 4 is repeated.

Step8: Next, the open termination is replaced with a short, and step 4 is repeated for this state of termination.

Step9: The short and $50 \mathrm{ohm}$ terminations at ports 5 and 6 are switched and step 4 is again repeated.

Step 10: The short termination is then removed, and the port is left open without any termination. This is the condition of offset open and step 4 is again repeated. The roles of ports 5 and 6 are now reversed and step 4 is again repeated. The noise powers measured in these steps are then imported into the calibration equations described in section 2 to determine $\mathbf{c}_{i}$ and its correlation with $\mathbf{c}_{e}$. This concludes the calibration of the measurement system.

With the measurement system thus calibrated, the noise parameters of a DUT can now be measured. The selected DUT for measurement is Wiltron $10 \mathrm{~dB}$ attenuator.

The noise parameters of the attenuator can easily be obtained from simulation, once its S-parameters are measured. The measured S-parameters were imported into Advanced Design System (ADS), a software from Keysight Technologies, as an $s 2 p$ data item and its noise parameters were obtained from noise simulation. The noise parameters thus obtained are used as the reference for the purpose of comparison with the noise parameters obtained via the 6-port noise wave correlator technique presented here.

\section{Results}

According to the Bosma Theory [30], the noise parameters of a passive device can be obtained from its Sparameters. This theory makes it possible for the noise parameters of a passive device to be determined from noise simulation once the S-parameters are available. We measured the S-parameters of the Wiltron 10-dB attenuator, and after importing same into Advanced Design Systems software, we simulated its noise parameters. This is the most ideal results the noise parameters of the 10-dB attenuator can approach. Thus by comparing the noise parameters results obtained using the measurement technique presented here, we are able to determine to what extent our technique is able to estimate the noise parameters of a sample device.

Figure 3 shows the results of the measured noise parameters of the Wiltron10-dB attenuator, which are 
obtained by converting the measured noise wave correlation matrix using well-known conversion formulae as discussed earlier. The agreement between the measured results via the 6-port network noise wave correlator technique, on one hand and that from the simulated results on the other are obviously very good. In the case of the minimum noise figure, $N F_{\text {min }}$, the mean value is $9.815 \mathrm{~dB}$ for the reference (simulated) data and 9.816 for the measured data obtained from the technique presented here. Furthermore, the measured results via the 6-port network noise wave correlator deviates from the simulated results by no more than $0.1 \mathrm{~dB}$. Notwithstanding the large values of the noise resistance, $R_{n}$, a mean of $151.63 \Omega$ for the 6-port network noise wave correlator technique and $150.476 \Omega$ for the simulated results, the measured data deviates from the simulated results by no more than $1.98 \%$. The two traces in the case of the optimum impedance, $Z_{\text {opt }}$ are barely distinguishable. All these underscore very good results from the 6-port noise wave correlator technique.

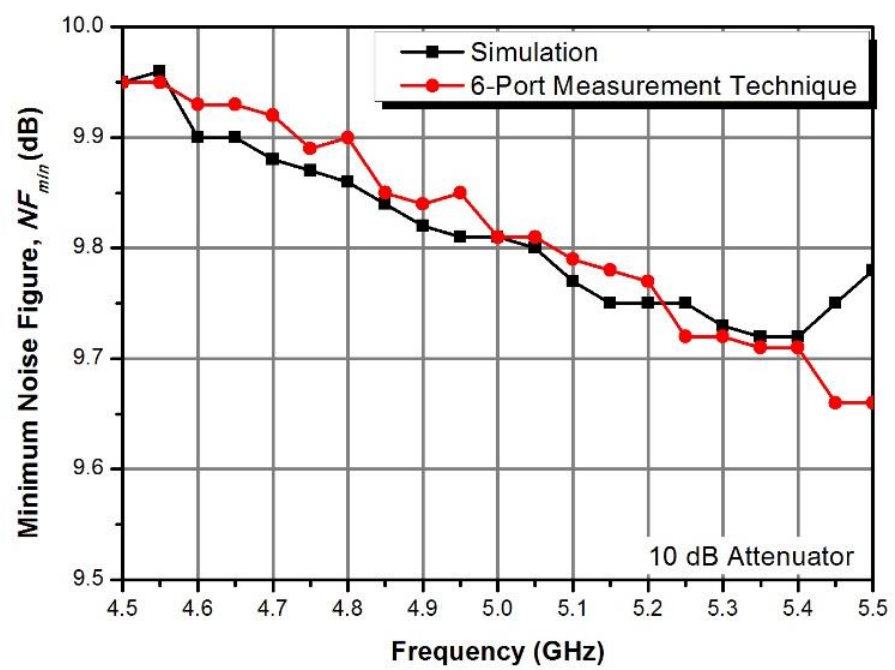

(a)

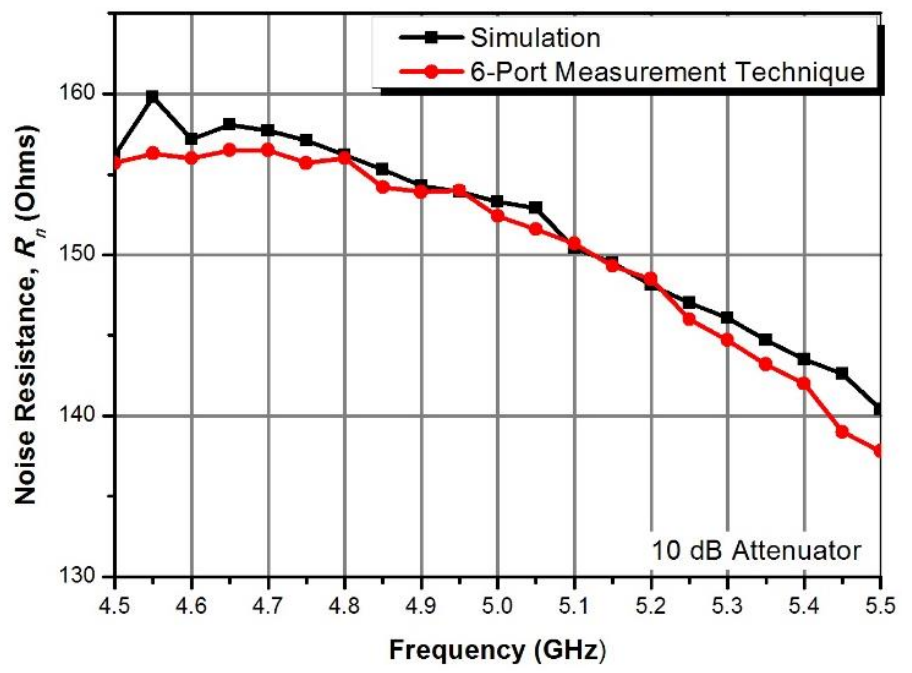

(b) 


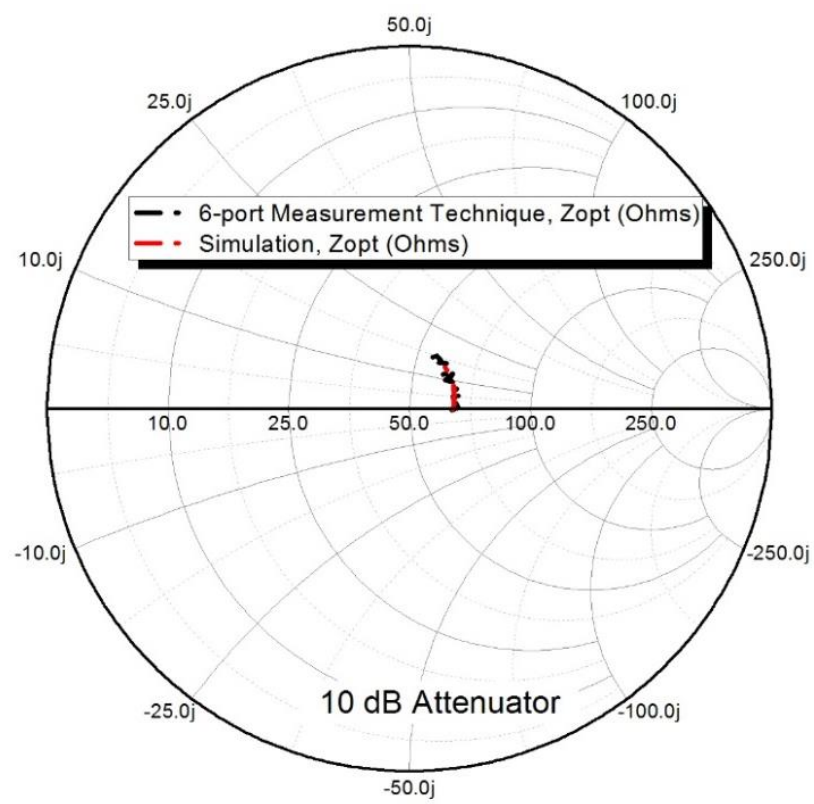

(c)

Fig.3. Noise Parameters of Wiltron $10 \mathrm{~dB}$ attenuator, (a) Minimu Noise Figure, $N F_{\min }$ (b) Noise Resistance, $R_{n}$ and (c) Optimum Impedance, $Z_{\text {opt }}$.

\section{Conclusions}

A formulation for the extraction of the noise wave correlation matrix with a simplified noise calibration of a designed 6-port network was presented. The simplified calibration procedure, which can be seen to be no more complicated a task than the calibration of a network analyzer for S-parameters measurement, applied to the designed six-port network enabled the extraction of the noise parameter of a $10 \mathrm{~dB}$ attenuator in a fairly straightforward manner. The only major cost in the measurement set up is that of the noise figure analyzer, and the rest of the components in the measurement set up can be obtained from any standard microwave lab, with the complement of off-the-shelve low noise amplifiers. Applying this 6-port noise wave correlator technique enables the extraction of the noise correlation matrix of a given DUT, and thus its noise parameters in a fairly fast, low cost and accurate manner.

\section{References}

[1] D. Middleton, "Statistical-physical models of Electromagnetic Interference," IEEE Trans. Electromagnetic Compatibility., vol. 19, no. 3, pp. 106-127, Aug. 1977.

[2] H. Rothe and W. Dahlke, "Theory of noisy four poles," Proc. IRE, vol. 44, pp. 811-818; Jun. 1956.

[3] R. Q. Lane, "The determination of device noise parameter," Proc. IEEE, vol. 57, pp. 1461-1462, Aug. 1969.

[4] M. S. Gupta, "Determination of the noise parameters of a linear 2-port," Electron. Lett., vol. 6, no. 17, pp. 543-544, Aug. 1970.

[5] M. Mitama and H. Katoh, "An improved computational method for noise parameter measurement," IEEE Trans. Microw. Theory Tech., vol. 27, no. 6, pp. 612-615, Jun. 1979. 
[6] G. Caruso and M. Sannino, "Computer-aided determination of microwave two-port noise parameters," IEEE Trans. Microw. Theory Tech., vol. 26, no. 9, pp. 639-642, Sept. 1978.

[7] G. I. Vasilescu, G. Alquie and M. Krim, "Exact computation of two-port noise parameters," Electron. Lett., vol. 25, no. 4, pp. 292-293, Feb. 1989.

[8] V. Adamian and A. Uhlir, "A novel procedure for receiver noise characterization," IEEE Trans. Instrum. and Meas., vol. 22, no. 2, pp. 181-182, Jun. 1973.

[9] J. Lange, "Noise characterization of linear two ports in terms of invariant parameters," IEEE J. Solid-State Circuits, vol. 2, no. 2, pp. 37-40, Jun. 1967.

[10] J. M. O'Callaghan and J. P. Mondal, "A vector approach for noise parameter fitting and selection of source admittances," IEEE Trans. Microw. Theory Tech, vol. 39, no. 8, pp. 1376-1382, Aug. 1991.

[11] A. C. Davidson, B. W. Bake, and E, Strid, "Accuracy Improvements in microwave noise parameter measurements," IEEE Trans. Microw. Theory Tech., vol. 37, no. 12, pp. 1973-1989, Dec. 1989.

[12] E. W. Strid, "Measurement of losses in noise-matching networks," IEEE Trans. Microw. Theory Tech., vol. 29, pp. 247-252, Mar. 1981.

[13] R. Meys, "A wave approach to the noise properties of linear microwave devices," IEEE Trans. Microw. Theory Tech., vol. 26, no. 1, pp. 34-37, Jan. 1978.

[14] P. Penfield, "Wave representation of amplifier noise," IRE Trans. Circuit Theory, vol. 9, pp. 84-86, Mar. 1962.

[15] S. W. Wedge and D. B. Rutledge, "Wave Techniques for Noise Modeling and Measurement," IEEE Trans. Microw. Theory Tech. vol. 40, no. 11, pp. 2004-2012, Nov. 1992.

[16] R. P. Hecken, "Analysis of linear noisy two-ports using scattering waves," IEEE Trans. Microw. Theory Tech. vol. 29, no. 10, pp. 997-1004, Oct. 1981.

[17] N. G. Kanaglekar, R. E. McIntosh, and W. E. Bryant, "Wave analysis of noise in interconnected multiport networks," IEEE Trans. Microw. Theory Tech., vol. 35, no. 2, pp. 112-115, Feb. 1987.

[18] J. A. Dobrowolski, "Noise power sensitivities and noise figure minimization of two-ports with any internal topology," IEEE Trans. Microw. Theory Tech., vol. 39, no. 1, pp. 136-140, Jan. 1991.

[19] S. Withington. "Scattered noise waves in microwave and mm-wave networks," Microwave J., vol. 32, pp. 169-178, Jun. 1989.

[20] S. W. Wedge, Computer-aided design of low noise microwave circuits, Ph.D. dissertation, California Institute of Technology, 1991.

[21] D. B. Rutledge and S. W. Wedge, Microwave six-port noise parameter analyzer: US patent, US 5170126 A, 1992

[22] J. A. Dobrowolski. "A CAD-oriented method for noise figure computation of two-ports with any internal topology," IEEE Trans. Microw. Theory Tech., vol. 37, no. 1, pp. 15-20, Jan. 1989.

[23] A. Lázaro, M. C. Maya, and L. Pradell, "Measurement of on-wafer transistor noise parameters without a tuner using unrestricted noise sources," Microwave J., vol. 45, no. 3, pp. 20-46, Mar. 2002.

[24] G. Dambrine, H. Cappy, F. Danneville, and A. Cappy, "A new method for on wafer noise measurement," IEEE Trans. Microwave Theory Tech., vol. 41, no. 3, pp. 375-381, Mar. 1993.

[25] F. Giannini, E. Bourdel, "A New Method to Extract Noise Parameters Based on a Frequency- and TimeDomain Analysis of Noise Power Measurements," IEEE Trans. Microw. Theory Tech. vol. 57, no. 2, pp. 261-267, Feb. 2008.

[26] T. Werling, E. Bourdel, D. Pasquet, and A. Boudiaf, "Determination of wave noise sources using spectral parametric modeling," IEEE Trans. Microw. Theory Tech., vol. 45, no. 12, pp. 2461-2467, Dec. 1997.

[27] G. F. Engen, and C. A. Hoer, "Thru-Reflect-Line: An Improved Technique for Calibrating the Dual SixPort Automatic Network Analyzer," IEEE Trans. Microw. Theory Tech., vol. 27, no. 12, pp. 987-992, Dec. 1979.

[28] A. -R. Ahmed, K. -W. Yeom, "An extraction of two-port noise parameters from measured noise powers using an extended six-port network", IEEE Trans on Microw. Theory and Tech., vol. 62, no. 10, pp. 24232434, Oct. 2014. 
[29] H. Hillbrand, P. H. Russer, "An efficient Method for Computer Aided Noise Analysis of Linear Amplifier Networks," IEEE Trans. on Circuits and Systems, vol. 23, no. 4, pp. 235-238, Apr. 1976.

[30] H. Bosma, "On the Theory of Linear Noisy Systems," Philips Res. Repts. Suppl., no. 10, 1967.

\section{Authors' Profiles}

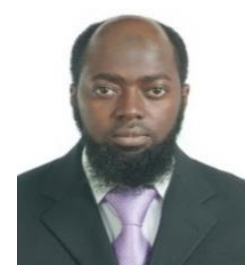

Abdul-Rahman Ahmed was born in Yeji, Ghana, in 1977. He received the MSc. Degree in Radio Systems Engineering from the University of Hull in 2005 and a Ph.D. degree in radio science and engineering from Chungnam National University, Daejeon, Korea in 2014. He has been a lecturer with the Department of Electrical and Electronic Engineering, Kwame Nkrumah University of Science and Technology, Kumasi, Ghana, since 2005. His current research interests are noise parameter measurement techniques, Microwave Circuit Design and Mobile Radio Propagation.

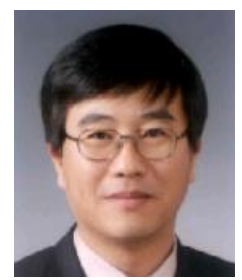

Kyung-Whan Yeom was born in Seoul, Korea, in 1957. He received the B.S. degree in electronics from Seoul National University, Seoul, Korea, in 1980, and the M.S. and Ph.D. degrees in electrical engineering from the Korea Advanced Institute of Science and Technology (KAIST), Daejeon, Korea, in 1982 and 1988, respectively. From 1985 to 1991, he was with LG Precision, as a Principal Engineer. He worked with the microwave integrated circuit (MIC) team as a Team Leader and was subsequently involved with the Military Electronics Division for electronic warfare (EW) equipment. From 1991 to 1995, he was with LTI, where he was involved with power-amplifier modules for analog cellular phones. In 1995, he joined Chungnam National University, Daejeon, Korea, as an Assistant Professor, and is currently a Professor with the Department of Radio Science and Engineering. His research interests are in the design of hybrid and monolithic microwave circuits and microwave systems. Prof. Yeom is a member of the Korean Institute of Electromagnetic Engineering and Science (KIEES) since 1995. He was the editor-in-chief of KIEES from 2004 to 2006. He was the recipient of the IR-52 Jang Youg-Sil Prize from the Ministry of Science and Technology (MOST) of Korea for his work on cellphone power amplifiers in 1994. He was also the recipient of the Academic Award of KIEES in 2004.

How to cite this paper: Abdul-Rahman Ahmed, Kyung-Whan Yeom,"A Low-Cost Noise Wave Correlator for Noise Parameters Extraction by Cold Noise Power Measurement", International Journal of Wireless and Microwave Technologies(IJWMT), Vol.6, No.5, pp.10-21, 2016.DOI: 10.5815/ijwmt.2016.05.02 